\title{
On Stability Analysis for Generalized Neural Networks with Time-Varying Delays
}

\author{
M. J. Park, ${ }^{1}$ O. M. Kwon, ${ }^{1}$ and E. J. Cha ${ }^{2}$ \\ ${ }^{1}$ School of Electrical Engineering, Chungbuk National University, 52 Naesudong-ro, Cheongju 361-763, Republic of Korea \\ ${ }^{2}$ Department of Biomedical Engineering, School of Medicine, Chungbuk National University, 52 Naesudong-ro, \\ Cheongju 361-763, Republic of Korea
}

Correspondence should be addressed to O. M. Kwon; madwind@chungbuk.ac.kr

Received 20 August 2014; Accepted 21 October 2014

Academic Editor: Jun Cheng

Copyright (C) 2015 M. J. Park et al. This is an open access article distributed under the Creative Commons Attribution License, which permits unrestricted use, distribution, and reproduction in any medium, provided the original work is properly cited.

This paper deals with the problem of stability analysis for generalized neural networks with time-varying delays. With a suitable Lyapunov-Krasovskii functional (LKF) and Wirtinger-based integral inequality, sufficient conditions for guaranteeing the asymptotic stability of the concerned networks are derived in terms of linear matrix inequalities (LMIs). By applying the proposed methods to two numerical examples which have been utilized in many works for checking the conservatism of stability criteria, it is shown that the obtained results are significantly improved comparing with the previous ones published in other literature.

\section{Introduction}

Neural networks have been successfully applied to various science and engineering problems such as pattern recognition, optimization, medical diagnosis, and image and signal processing [1-4]. In addition to this, the model of neural networks can take a variety of forms such as bidirectional associative memory (BAM) [5] and Cohen-Grossberg [6] neural networks. Therefore, the stability analysis of neural networks has been extensively studied because the application of neural networks heavily depends on the dynamic behavior of its equilibrium points and it is a prerequisite job to check the stability of the concerned networks. Recently, a model of neural networks having time-varying delays has been considered and its asymptotic stability has been extensively investigated due to the fact that the occurrence of time delays, which are caused by the inherent communication time among the neurons and the finite switching speed of amplifiers in hardware implementation of the networks, can harm the system performance and stability. With this regard, a lot of weight has been placed on the stability analysis for local field and static neural networks with time-varying delays. For local field neural networks, a new activation condition which has not been considered was proposed to reduce the conservatism of stability criteria in [7]. Two delaypartitioning approaches were utilized to get further enhanced delay-dependent stability criteria for neural networks with time-varying delays in [8-11]. Very recently, a new augmented Lyapunov-Krasovskii functional was proposed to enhance the feasible region of stability condition of delayed neural networks [12]. For static neural networks, improved delaydependent stability criteria were presented in $[13,14]$. By constructing an augmented Lyapunov-Krasovskii functional, both delay-independent and delay-dependent stability criteria were proposed in [15] with some new techniques. The robust global asymptotic stability of generalized static neural networks with linear fractional uncertainties and time-varying delays was proposed in [16] within a novel input-output framework. Very recently, interval time-varying delays were considered to investigate the problem of delaydependent stability of static neural networks [17].

The main issue of delay-dependent stability analysis for dynamic systems with time-delays is to enhance the feasible region comparing with the existing results. In delaydependent stability analysis, maximum delay bounds for guaranteeing the asymptotic stability have been considered 
as one of the index for checking the conservatism of stability condition. The reduction of conservatism in delay-dependent stability criteria mainly depends on the construction of LKF and the utilization of some techniques to estimate the timederivative of LKF. In LKF point of view, discretized form [18], triple integral form [19-21], and augmented LKF in [12, 22] and $[23,24]$ have been proposed to reduce the conservatism of stability condition. In techniques for estimating the timederivative of LKFs, Park's inequality [25], Jensen inequality [18], model transformation [26, 27], free-weighting matrices techniques [28, 29], convex combination technique [30], reciprocally convex optimization [31], and the Wirtingerbased integral inequality [32] are remarkable results which promotes the development of delay-dependent stability analysis. Among the techniques in estimating the time-derivative of LKFs, Wirtinger-based integral inequality, which provides more tighter lower bound of integral terms than Jensen's inequality, has been applied to stability analysis of delayed neural networks $[33,34]$ very recently. However, in stability analysis for neural networks with time-varying delays, the application of the Wirtinger-based integral inequality with an augmented LKF has not been fully investigated yet and thus there is still room for further improvements on the reduction of conservatism.

With the motivation mentioned above, the problem of stability analysis for generalized neural network with timevarying delays is investigated in this paper. Inspired by the works $[35,36]$, a generalized neural network which contains the static neural networks and the local field neural networks as special cases is considered in this work. Thus, the general model of neural networks used in this work is a superordinate concept to the model utilized in most literature including the works [37-41]. In Lemma 3, a modified Wirtinger-based integral inequality which changes the intervals of integral terms will be introduced as Lemma 3. Then, by constructing a suitable augmented Lyapunov-Krasovskii functional and utilizing Lemma 3, an improved stability condition such that the considered neural networks are asymptotically stable is derived in terms of linear matrix inequalities (LMIs). When the information about the time-derivative of time-varying delays is unknown, a stability condition will be presented as Corollary 10. The advantage and superiority of the main results will be shown via two numerical examples which have been utilized in many previous works to check the conservatism of stability criteria.

Notation. $\mathbb{R}^{n}$ and $\mathbb{R}^{m \times n}$ denote the $n$-dimensional Euclidean space with vector norm $\|\cdot\|$ and the set of all $m \times n$ real matrices, respectively. $\mathbb{S}^{n}$ and $\mathbb{S}_{+}^{n}$ are the sets of symmetric and positive definite $n \times n$ matrices, respectively. $I_{n}, 0_{n}$, and $0_{m \cdot n}$ denote $n \times n$ identity matrix, $n \times n$ and $m \times n$ zero matrices, respectively. $X>0(<0)$ means symmetric positive (negative) definite matrix. $X^{\perp}$ stands for a basis for the null space of $X$. $\operatorname{diag}\{\cdots\}$ represents the block diagonal matrix. For any square matrix $X$ and any vectors $x_{i}$, respectively, we define $\operatorname{Sym}\{X\}=$ $X+X^{T}$ and $\operatorname{col}\left\{x_{1}, x_{2}, \ldots, x_{n}\right\}=\left[\begin{array}{llll}x_{1}^{T} & x_{2}^{T} & \cdots & x_{n}^{T}\end{array}\right]^{T} \cdot X_{[f(t)]}$ means that the elements of matrix $X_{[f(t)]}$ include the scalar value of $f(t)$ affinely.

\section{Problem Statement and Preliminaries}

Consider the following generalized neural networks with time-varying delays:

$$
\dot{y}(t)=-A y(t)+W_{0} g\left(W_{2} y(t)\right)+W_{1} g\left(W_{2} y(t-h(t))\right)+b,
$$

where $n$ denotes the number of neurons in a neural network, $y(t)=\left[y_{1}(t), \ldots, y_{n}(t)\right]^{T} \in \mathbb{R}^{n}$ is the state vector of neuron, $g(\cdot)=\left[g_{1}(\cdot), \ldots, g_{n}(\cdot)\right]^{T} \in \mathbb{R}^{n}$ denotes the activation function of neuron, $A=\operatorname{diag}\left\{a_{i}\right\} \in \mathbb{R}^{n \times n}>0$ is the system matrix, $W_{j} \in$ $\mathbb{R}^{n \times n}(j=0,1,2)$ are the interconnection weight matrices, and $b=\left[b_{1}, \ldots, b_{n}\right]^{T} \in \mathbb{R}^{n}$ is a constant input vector.

$h(t)$ is the time-varying delay satisfying

$$
0 \leq h(t) \leq h_{U}, \quad \dot{h}(t) \leq h_{D}
$$

where $h_{U}$ is a known positive scalar and $h_{D}$ is any constant one.

The activation functions of neuron satisfy the following assumption.

Assumption 1. The activation functions of neuron $g_{i}(\cdot)(i=$ $1, \ldots, n)$ with $g_{i}(0)=0$ are continuous, bounded and satisfy

$$
k_{i}^{-} \leq \frac{g_{i}\left(s_{1}\right)-g_{i}\left(s_{2}\right)}{s_{1}-s_{2}} \leq k_{i}^{+}, \quad s_{1}, s_{2} \in \mathbb{R}, s_{1} \neq s_{2},
$$

where $k_{i}^{+}$and $k_{i}^{-}$are known real constants.

Remark 2. In Assumption $1, k_{i}^{+}$and $k_{i}^{-}$can be allowed to be positive, negative, or zero. As mentioned in [40], Assumption 1 describes the class of globally Lipschitz continuous and monotone nondecreasing activation when $k_{i}^{-}=0$ and $k_{i}^{+}>0$. And the class of globally Lipschitz continuous and monotone increasing activation functions can be described when $k_{i}^{+}>k_{i}^{-}>0$.

For analyzing stability of the neural networks (1), the equilibrium point $y^{*}=\left[y_{1}^{*}, \ldots, y_{n}^{*}\right]^{T}$ whose uniqueness has been reported in [41] is shifted to the origin by utilizing the transformation $x(\cdot)=y(\cdot)-y^{*}$, which leads the system (1) to the following form:

$$
\dot{x}(t)=-A x(t)+W_{0} f\left(W_{2} x(t)\right)+W_{1} f\left(W_{2} x(t-h(t))\right),
$$

where $x(t)=\left[x_{1}(t), \ldots, x_{n}(t)\right]^{T} \in \mathbb{R}^{n}$ is the state vector of the transformed system, $W_{2}=\left[W_{2,1}^{T}, \ldots, W_{2, n}^{T}\right]^{T}$ with $W_{2, i} \in \mathbb{R}^{1 \times n}, f\left(W_{2} x(t)\right)=\left[f_{1}\left(W_{2,1} x(t)\right), \ldots, f_{n}\left(W_{2, n} x(t)\right)\right]^{T}$, and $f_{j}\left(W_{2, j} x(t)\right)=g_{j}\left(W_{2, j} x(t)+W_{2, j} y^{*}\right)-g_{j}\left(W_{2, j} y^{*}\right)$ with $f_{j}(0)=0(j=1, \ldots, n)$.

It should be noted that the activation functions $f_{i}(\cdot)(i=$ $1, \ldots, n)$ satisfy the following condition [42]:

$$
\begin{gathered}
k_{i}^{-} \leq \frac{f_{i}\left(W_{2, i} u\right)-f_{i}\left(W_{2, i} v\right)}{W_{2, i} u-W_{2, i} v} \leq k_{i}^{+}, \quad u, v \in \mathbb{R}^{n}, u \neq v, \\
k_{i}^{-} \leq \frac{f_{i}\left(W_{2, i} u\right)}{W_{2, i} u} \leq k_{i}^{+}, \quad \forall u \neq 0, i=1, \ldots, n .
\end{gathered}
$$


The aim of this paper is to investigate the delay-dependent stability analysis of system (4) which will be introduced in next section. Before deriving our main results, the following lemmas will be utilized in deriving the main results.

Lemma 3 (Modified Wirtinger-based integral inequality). Consider a given matrix $R>0$. Then, for all continuous function $\omega$ in $[a, b] \rightarrow \mathbb{R}^{n}$, the following inequality holds:

$$
\begin{aligned}
\int_{a}^{b} \omega^{T}(s) & R \omega(s) d s \\
\geq & \frac{1}{b-a}\left(\int_{a}^{b} \omega(s) d s\right)^{T} R\left(\int_{a}^{b} \omega(s) d s\right) \\
& +\frac{3}{b-a}\left(\int_{a}^{b} \omega(s) d s-\frac{2}{b-a} \int_{a}^{b} \int_{s}^{b} \omega(u) d u d s\right)^{T} \\
\quad \times & R\left(\int_{a}^{b} \omega(s) d s-\frac{2}{b-a} \int_{a}^{b} \int_{s}^{b} \omega(u) d u d s\right) .
\end{aligned}
$$

Proof. From the term $\int_{a}^{b} \omega(s) d s-(2 /(b-a)) \int_{a}^{b} \int_{a}^{s} \omega(u) d u d s$ in the original Wirtinger-based integral inequality [32], since

$$
\begin{aligned}
& \int_{a}^{b} \omega(s) d s-\frac{2}{b-a} \int_{a}^{b} \int_{a}^{s} \omega(u) d u d s \\
& =\int_{a}^{b} \omega(s) d s-\frac{2}{b-a} \int_{a}^{b}\left(\int_{a}^{b} \omega(u) d u-\int_{s}^{b} \omega(u) d u\right) d s \\
& =\int_{a}^{b} \omega(s) d s \\
& \quad-\frac{2}{b-a} \iint_{a}^{b} \omega(u) d u d s+\frac{2}{b-a} \int_{a}^{b} \int_{s}^{b} \omega(u) d u d s \\
& =-\int_{a}^{b} \omega(s) d s+\frac{2}{b-a} \int_{a}^{b} \int_{s}^{b} \omega(u) d u d s,
\end{aligned}
$$

the inequality (7) holds.

Lemma 4 (see [43]). Let $\zeta \in \mathbb{R}^{n}$, $\Phi=\Phi^{T} \in \mathbb{R}^{n \times n}$, and $B \in$ $\mathbb{R}^{m \times n}$ such that $\operatorname{rank}(B)<n$. Then, the following statements are equivalent:

(1) $\zeta^{T} \Phi \zeta<0, B \zeta=0, \zeta \neq 0$,

(2) $\left(B^{\perp}\right)^{T} \Phi B^{\perp}<0$, where $B^{\perp}$ is a right orthogonal complement of $B$.

Lemma 5 (see [44]). For symmetric appropriately dimensional matrices $\Omega>0, \Xi$, matrix $\Lambda$, the following two statements are equivalent:

(1) $\Xi-\Lambda^{T} \Omega \Lambda<0$,

(2) there exists a matrix of appropriate dimension $\Psi$ such that

$$
\left[\begin{array}{cc}
\Xi+\Lambda^{T} \Psi+\Psi^{T} \Lambda & \Psi^{T} \\
\Psi & -\Omega
\end{array}\right]<0 .
$$

\section{Main Results}

In this section, two delay-dependent stability criteria for system (4) will be proposed. For the sake of simplicity of matrix and vector representation, $e_{i}(i=1,2, \ldots, 12) \epsilon$ $\mathbb{R}^{12 n \times n}$ which will be used in Theorem 6 and Corollary 10 are defined as block entry matrices. (e.g., $e_{3}=\left[0_{n \cdot 2 n}, I_{n}, 0_{n \cdot 9 n}\right]^{T}$ ). The other notations for some vectors and matrices are defined in the appendix.

Now, the following theorem is given as a main result.

Theorem 6. For given scalars $h_{U}>0, h_{D}$, and diagonal matrices $K_{m}=\operatorname{diag}\left\{k_{1}^{-}, \ldots, k_{n}^{-}\right\}$and $K_{p}=\operatorname{diag}\left\{k_{1}^{+}, \ldots, k_{n}^{+}\right\}$, system (4) is asymptotically stable for $0 \leq h(t) \leq h_{U}$ and $\dot{h}(t) \leq h_{D}$, if there exist matrices $\Lambda=\operatorname{diag}\left\{\lambda_{1}, \ldots, \lambda_{n}\right\} \in \mathbb{S}_{+}^{n}$, $\Delta=\operatorname{diag}\left\{\delta_{1}, \ldots, \delta_{n}\right\} \in \mathbb{S}_{+}^{n}, H_{i}=\operatorname{diag}\left\{h_{1 i}, \ldots, h_{n i}\right\} \in \mathbb{S}_{+}^{n}(i=$ $1,2, \ldots, 5), \mathscr{R} \in \mathbb{S}_{+}^{3 n}, \mathcal{N} \in \mathbb{S}_{+}^{3 n}, \mathscr{G}_{1} \in \mathbb{S}_{+}^{3 n}, G_{2} \in \mathbb{S}_{+}^{n}, \mathbb{Q} \in \mathbb{S}_{+}^{3 n}$, $\mathcal{S} \in \mathbb{R}^{6 n \times 6 n}, F \in \mathbb{R}^{15 n \times 11 n}, Z_{i} \in \mathbb{S}^{n}(i=1,2)$ satisfying the following LMIs:

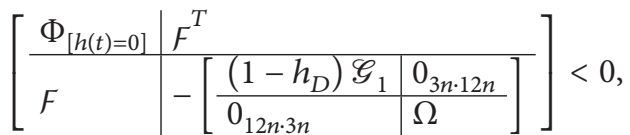

$$
\begin{aligned}
& {\left[\begin{array}{l|l|l}
\Phi_{\left[h(t)=h_{U}\right]} & F^{T} \\
\hline F & -\left[\begin{array}{ll}
\left(1-h_{D}\right) \mathscr{G}_{1} & 0_{3 n \cdot 12 n} \\
\hline 0_{12 n \cdot 3 n} & \Omega
\end{array}\right]<0 .
\end{array}\right.}
\end{aligned}
$$

Proof. Let us consider the following candidate for the appropriate Lyapunov-Krasovskii functional:

$$
V(t)=V_{1}(t)+V_{2}(t)+V_{3}(t)+V_{4}(t)+V_{5}(t),
$$

where

$$
\begin{aligned}
& V_{1}(t)=\left[\begin{array}{c}
x(t) \\
x\left(t-h_{U}\right) \\
\int_{t-h_{U}}^{t} x(s) d s
\end{array}\right]^{T} \mathscr{R}\left[\begin{array}{c}
x(t) \\
x\left(t-h_{U}\right) \\
\int_{t-h_{U}}^{t} x(s) d s
\end{array}\right], \\
& V_{2}(t)=2 \sum_{i=1}^{n}\left(\lambda_{i} \int_{0}^{W_{2, i} x(t)}\left(f_{i}(s)-k_{i}^{-} s\right) d s\right. \\
& \left.+\delta_{i} \int_{0}^{W_{2, i} x(t)}\left(k_{i}^{+} s-f_{i}(s)\right) d s\right), \\
& V_{3}(t)=\int_{t-h_{U}}^{t}\left[\begin{array}{c}
x(s) \\
\dot{x}(s) \\
f\left(W_{2} x(s)\right)
\end{array}\right]^{T} \mathcal{N}\left[\begin{array}{c}
x(s) \\
\dot{x}(s) \\
f\left(W_{2} x(s)\right)
\end{array}\right] d s, \\
& V_{4}(t)=\int_{t-h(t)}^{t}\left[\begin{array}{c}
x(s) \\
\int_{s}^{t} \dot{x}(u) d u \\
\int_{s}^{t} x(u) d u
\end{array}\right]^{T} \mathscr{G}_{1}\left[\begin{array}{c}
x(s) \\
\int_{s}^{t} \dot{x}(u) d u \\
\int_{s}^{t} x(u) d u
\end{array}\right] d s \\
& +\int_{t-h(t)}^{t} f^{T}\left(W_{2} x(s)\right) G_{2} f\left(W_{2} x(s)\right) d s, \\
& V_{5}(t)=h_{U} \int_{t-h_{U}}^{t} \int_{s}^{t}\left[\begin{array}{c}
\dot{x}(u) \\
x(u) \\
\int_{u}^{t} \dot{x}(v) d v
\end{array}\right]^{T} Q\left[\begin{array}{c}
\dot{x}(u) \\
x(u) \\
\int_{u}^{t} \dot{x}(v) d v
\end{array}\right] d u d s .
\end{aligned}
$$


The results of the time-derivative of $V_{i}(t)(i=1,2,3)$ are as follows:

$$
\begin{aligned}
& \dot{V}_{1}(t)=2\left[\begin{array}{c}
x(t) \\
x\left(t-h_{U}\right) \\
h(t) \nu_{1}(t)+\left(h_{U}-h(t)\right) v_{2}(t)
\end{array}\right]^{T}
\end{aligned}
$$

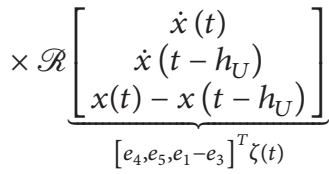

$$
\begin{aligned}
& =\zeta^{T}(t) \Xi_{1[h(t)]} \zeta(t), \\
& \dot{V}_{2}(t)=2\left[f\left(W_{2} x(t)\right)-K_{m} W_{2} x(t)\right]^{T} \Lambda W_{2} \dot{x}(t) \\
& +2\left[K_{p} W_{2} x(t)-f\left(W_{2} x(t)\right)\right]^{T} \Delta W_{2} \dot{x}(t) \\
& =\zeta^{T}(t) \Xi_{2} \zeta(t),
\end{aligned}
$$$$
\dot{V}_{3}(t)=\left[\begin{array}{c}
x(t) \\
\dot{x}(t) \\
f\left(W_{2} x(t)\right)
\end{array}\right]^{T} \mathcal{N}\left[\begin{array}{c}
x(t) \\
\dot{x}(t) \\
f\left(W_{2} x(t)\right)
\end{array}\right]
$$$$
-\left[\begin{array}{c}
x\left(t-h_{U}\right) \\
\dot{x}\left(t-h_{U}\right) \\
f\left(W_{2} x\left(t-h_{U}\right)\right)
\end{array}\right]^{T} \mathcal{N}\left[\begin{array}{c}
x\left(t-h_{U}\right) \\
\dot{x}\left(t-h_{U}\right) \\
f\left(W_{2} x\left(t-h_{U}\right)\right)
\end{array}\right]
$$$$
=\zeta^{T}(t) \Xi_{3} \zeta(t) \text {. }
$$

By applying Leibniz integral rule to $\dot{V}_{4}(t)$, it follows that

$$
\dot{V}_{4}(t)
$$

$$
=\left.\left[\begin{array}{l}
x(s) \\
\int_{s}^{t} \dot{x}(u) d u \\
\int_{s}^{t} x(u) d u
\end{array}\right]^{T} \mathscr{G}_{1}\left[\begin{array}{c}
x(s) \\
\int_{s}^{t} \dot{x}(u) d u \\
\int_{s}^{t} x(u) d u
\end{array}\right]\right|_{s=t} \times \frac{d}{d t}(t)
$$

$$
\begin{aligned}
& -\left.\left[\begin{array}{c}
x(s) \\
\int_{s}^{t} \dot{x}(u) d u \\
\int_{s}^{t} x(u) d u
\end{array}\right]^{T} \mathscr{G}_{1}\left[\begin{array}{c}
x(s) \\
\int_{s}^{t} \dot{x}(u) d u \\
\int_{s}^{t} x(u) d u
\end{array}\right]\right|_{s=t-h(t)} \\
& \times \frac{d}{d t}(t-h(t)) \\
& +\int_{t-h(t)}^{t} \frac{\partial}{\partial t}\left(\left[\begin{array}{c}
x(s) \\
\int_{s}^{t} \dot{x}(u) d u \\
\int_{s}^{t} x(u) d u
\end{array}\right]^{T} \mathscr{G}_{1}\left[\begin{array}{c}
x(s) \\
\int_{s}^{t} \dot{x}(u) d u \\
\int_{s}^{t} x(u) d u
\end{array}\right]\right) d s \\
& +f^{T}\left(W_{2} x(t)\right) G_{2} f\left(W_{2} x(t)\right) \\
& \text { - }(1-\dot{h}(t)) f^{T}\left(W_{2} x(t-h(t))\right) \\
& \times G_{2} f\left(W_{2} x(t-h(t))\right) \\
& \leq\left[\begin{array}{c}
x(s) \\
0_{n \cdot 1} \\
0_{n \cdot 1}
\end{array}\right]^{T} \mathscr{G}_{1}\left[\begin{array}{c}
x(s) \\
0_{n \cdot 1} \\
0_{n \cdot 1}
\end{array}\right] \\
& -\left(1-h_{D}\right)\left[\begin{array}{c}
x(t-h(t)) \\
\int_{t-h(t)}^{t} \dot{x}(u) d u \\
\int_{t-h(t)}^{t} x(u) d u
\end{array}\right]^{T} \mathscr{G}_{1}\left[\begin{array}{c}
x(t-h(t)) \\
\int_{t-h(t)}^{t} \dot{x}(u) d u \\
\int_{t-h(t)}^{t} x(u) d u
\end{array}\right] \\
& +2\left[\begin{array}{c}
h(t) v_{1}(t) \\
h(t)\left(x(t)-v_{1}(t)\right) \\
h(t) v_{1}(t)
\end{array}\right]^{T} \mathscr{G}_{1}\left[\begin{array}{c}
0_{n \cdot 1} \\
\dot{x}(t) \\
x(t)
\end{array}\right] \\
& +f^{T}\left(W_{2} x(t)\right) G_{2} f\left(W_{2} x(t)\right) \\
& -\left(1-h_{D}\right) f^{T}\left(W_{2} x(t-h(t))\right) G_{2} f\left(W_{2} x(t-h(t))\right) \\
& =\zeta^{T}(t)\left(\Xi_{4[h(t)]}-\left(1-h_{D}\right) \Psi_{[h(t)]}^{T} \mathscr{G}_{1} \Psi_{[h(t)]}\right) \zeta(t) .
\end{aligned}
$$

Considering Leibniz integral rule and the two zero equalities inspired by the work of [45] to $\dot{V}_{5}(t)$ leads to

$$
\begin{aligned}
\dot{V}_{5}(t)+ & \underbrace{\left\{\begin{array}{l}
x^{T}(t)\left(h_{U} Z_{1}\right) x(t)-x^{T}(t-h(t))\left(h_{U} Z_{1}\right) x(t-h(t)) \\
-h_{U} \int_{t-h(t)}^{t}\left[\begin{array}{l}
x(t) \\
\dot{x}(t)
\end{array}\right]^{T}\left[\begin{array}{cc}
0_{n} & Z_{1} \\
Z_{1} & 0_{n}
\end{array}\right]\left[\begin{array}{l}
x(t) \\
\dot{x}(t)
\end{array}\right] d s
\end{array}\right]}_{0} \\
+ & \left\{\begin{array}{l}
x^{T}(t-h(t))\left(h_{U} Z_{2}\right) x(t-h(t))-x^{T}\left(t-h_{U}\right)\left(h_{U} Z_{2}\right) x\left(t-h_{U}\right) \\
-h_{U} \int_{t-h_{U}}^{t-h(t)}\left[\begin{array}{l}
x(t) \\
\dot{x}(t)
\end{array}\right]^{T}\left[\begin{array}{cc}
0_{n} & Z_{2} \\
Z_{2} & 0_{n}
\end{array}\right]\left[\begin{array}{l}
x(t) \\
\dot{x}(t)
\end{array}\right] d s
\end{array}\right\}
\end{aligned}
$$




$$
\begin{aligned}
& =-h_{U} \int_{t-h_{U}}^{t}\left[\begin{array}{c}
\dot{x}(s) \\
x(s) \\
\int_{s}^{t} \dot{x}(u) d u
\end{array}\right]^{T} Q\left[\begin{array}{c}
\dot{x}(s) \\
x(s) \\
\int_{s}^{t} \dot{x}(u) d u
\end{array}\right] d s \\
& -h_{U} \int_{t-h(t)}^{t}\left[\begin{array}{c}
x(t) \\
\dot{x}(t)
\end{array}\right]^{T}\left[\begin{array}{cc}
0_{n} & Z_{1} \\
Z_{1} & 0_{n}
\end{array}\right]\left[\begin{array}{c}
x(t) \\
\dot{x}(t)
\end{array}\right] d s \\
& -h_{U} \int_{t-h_{U}}^{t-h(t)}\left[\begin{array}{c}
x(t) \\
\dot{x}(t)
\end{array}\right]^{T}\left[\begin{array}{cc}
0_{n} & Z_{2} \\
Z_{2} & 0_{n}
\end{array}\right]\left[\begin{array}{c}
x(t) \\
\dot{x}(t)
\end{array}\right] d s \\
& +h_{U} \int_{t-h_{U}}^{t}\left(\left[\begin{array}{c}
\dot{x}(t) \\
x(t) \\
0_{n \cdot 1}
\end{array}\right]^{T} Q\left[\begin{array}{c}
\dot{x}(t) \\
x(t) \\
0_{n \cdot 1}
\end{array}\right]+2 \int_{s}^{t}\left[\begin{array}{c}
\dot{x}(u) \\
x(u) \\
\int_{u}^{t} \dot{x}(v) d v
\end{array}\right]^{T} Q\left[\begin{array}{c}
0_{n \cdot 1} \\
0_{n \cdot 1} \\
\dot{x}(t)
\end{array}\right] d u\right) d s \\
& +x^{T}(t)\left(h_{U} Z_{1}\right) x(t)-x^{T}(t-h(t))\left(h_{U} Z_{1}\right) x(t-h(t)) \\
& +x^{T}(t-h(t))\left(h_{U} Z_{2}\right) x(t-h(t))-x^{T}\left(t-h_{U}\right)\left(h_{U} Z_{2}\right) x\left(t-h_{U}\right) \\
& =h_{U}^{2}\left[\begin{array}{c}
\dot{x}(t) \\
x(t) \\
0_{n \cdot 1}
\end{array}\right]^{T} Q\left[\begin{array}{c}
\dot{x}(t) \\
x(t) \\
0_{n \cdot 1}
\end{array}\right] \\
& +2 h_{U} \underbrace{\left[\begin{array}{c}
h_{U} x(t)-h(t) v_{1}(t)-\left(h_{U}-h(t)\right) v_{2}(t) \\
h(t) v_{3}(t)+\left(h_{U}-h(t)\right) v_{4}(t) \\
\left(\frac{h_{U}^{2}}{2}\right) x(t)-h(t) v_{3}(t)-\left(h_{U}-h(t)\right) v_{4}(t)
\end{array}\right]^{T}}_{\Pi_{[h(t)]}^{T}} Q\left[\begin{array}{c}
0_{n \cdot 1} \\
0_{n \cdot 1} \\
\dot{x}(t)
\end{array}\right] \\
& +x^{T}(t)\left(h_{U} Z_{1}\right) x(t)-x^{T}(t-h(t))\left(h_{U} Z_{1}\right) x(t-h(t)) \\
& +x^{T}(t-h(t))\left(h_{U} Z_{2}\right) x(t-h(t))-x^{T}\left(t-h_{U}\right)\left(h_{U} Z_{2}\right) x\left(t-h_{U}\right) \\
& -h_{U} \int_{t-h(t)}^{t}\left[\begin{array}{c}
\dot{x}(s) \\
x(s) \\
\int_{s}^{t} \dot{x}(u) d u
\end{array}\right]_{\mathbf{Q}_{1}}^{\left(\mathcal{Q}+\left[\begin{array}{ccc}
0_{n} & Z_{1} & 0_{n} \\
Z_{1} & 0_{n} & 0_{n} \\
0_{n} & 0_{n} & 0_{n}
\end{array}\right]\right)}\left[\begin{array}{c}
\dot{x}(s) \\
x(s) \\
\int_{s}^{t} \dot{x}(u) d u
\end{array}\right] d s \\
& -h_{U} \int_{t-h_{U}}^{t-h(t)}\left[\begin{array}{c}
\dot{x}(s) \\
x(s) \\
\int_{s}^{t} \dot{x}(u) d u
\end{array}\right]^{T} \underbrace{\left(\underline{Q}+\left[\begin{array}{ccc}
0_{n} & Z_{2} & 0_{n} \\
Z_{2} & 0_{n} & 0_{n} \\
0_{n} & 0_{n} & 0_{n}
\end{array}\right]\right)}_{\mathbf{Q}_{2}}\left[\begin{array}{c}
\dot{x}(s) \\
x(s) \\
\int_{s}^{t} \dot{x}(u) d u
\end{array}\right] d s,
\end{aligned}
$$

where $Z_{1}$ and $Z_{2}$ are any symmetric matrices.

It should be noted that

$$
\begin{aligned}
& \frac{2}{h(t)} \int_{t-h(t)}^{t} \int_{s}^{t} \int_{u}^{t} \dot{x}(v) d v d u d s \\
& \quad=\frac{2}{h(t)} \int_{t-h(t)}^{t} \int_{s}^{t}(x(t)-x(u)) d u d s \\
& \quad=\frac{2}{h(t)}\left(\frac{h^{2}(t)}{2} x(t)-\int_{t-h(t)}^{t} \int_{s}^{t} x(u) d u d s\right) \\
& \quad=h(t) x(t)-\frac{2}{h(t)} \int_{t-h(t)}^{t} \int_{s}^{t} x(u) d u d s .
\end{aligned}
$$




$$
\begin{aligned}
& \times \mathbf{Q}_{1}\left(\int_{t-h(t)}^{t}\left[\begin{array}{c}
\dot{x}(s) \\
x(s) \\
\int_{s}^{t} \dot{x}(u) d u
\end{array}\right] d s\right) \\
& -\frac{3 h_{U}}{h(t)}\left(\int_{t-h(t)}^{t}\left[\begin{array}{c}
\dot{x}(s) \\
x(s) \\
\int_{s}^{t} \dot{x}(u) d u
\end{array}\right] d s\right. \\
& \left.-\frac{2}{h(t)} \int_{t-h(t)}^{t} \int_{s}^{t}\left[\begin{array}{c}
\dot{x}(s) \\
x(s) \\
\int_{s}^{t} \dot{x}(u) d u
\end{array}\right] d u d s\right)^{T} \mathbf{Q}_{1} \\
& \times\left(\int_{t-h(t)}^{t}\left[\begin{array}{c}
\dot{x}(s) \\
x(s) \\
\int_{s}^{t} \dot{x}(u) d u
\end{array}\right] d s\right. \\
& \left.-\frac{2}{h(t)} \int_{t-h(t)}^{t} \int_{s}^{t}\left[\begin{array}{c}
\dot{x}(s) \\
x(s) \\
\int_{s}^{t} \dot{x}(u) d u
\end{array}\right] d u d s\right) \\
& =-\frac{h_{U}}{h(t)}\left[\begin{array}{c}
x(t)-x(t-h(t)) \\
h(t) v_{1}(t) \\
h(t)\left(x(t)-v_{1}(t)\right)
\end{array}\right]^{T} \\
& \times \mathbf{Q}_{1}\left[\begin{array}{c}
x(t)-x(t-h(t)) \\
h(t) v_{1}(t) \\
h(t)\left(x(t)-v_{1}(t)\right)
\end{array}\right] \\
& -\frac{h_{U}}{h(t)}\left[\begin{array}{c}
-x(t)-x(t-h(t))+2 v_{1}(t) \\
h(t) v_{1}(t)-2 v_{3}(t) \\
2 v_{3}(t)-h(t) v_{1}(t)
\end{array}\right]^{T} \\
& \times\left(3 \mathbf{Q}_{1}\right)\left[\begin{array}{c}
-x(t)-x(t-h(t))+2 v_{1}(t) \\
h(t) v_{1}(t)-2 v_{3}(t) \\
2 v_{3}(t)-h(t) \nu_{1}(t)
\end{array}\right] \\
& =-\frac{h_{U}}{h(t)} \zeta^{T}(t) \Upsilon_{1[h(t)]}^{T}\left[\begin{array}{cc}
\mathbf{Q}_{1} & 0_{3 n} \\
0_{3 n} & 3 \mathbf{Q}_{1}
\end{array}\right] \Upsilon_{1[h(t)]} \zeta(t) \text {. }
\end{aligned}
$$

With the similar process presented in (18), it can be obtained that

$$
\begin{gathered}
-h_{U} \int_{t-h_{U}}^{t-h(t)}\left[\begin{array}{c}
\dot{x}(s) \\
x(s) \\
\int_{s}^{t} \dot{x}(u) d u
\end{array}\right]^{T} \mathbf{Q}_{2}\left[\begin{array}{c}
\dot{x}(s) \\
x(s) \\
\int_{s}^{t} \dot{x}(u) d u
\end{array}\right] d s \\
\leq-\frac{h_{U}}{h_{U}-h(t)} \zeta^{T}(t) \Upsilon_{2[h(t)]}^{T}\left[\begin{array}{cc}
\mathbf{Q}_{2} & 0_{3 n} \\
0_{3 n} & 3 \mathbf{Q}_{2}
\end{array}\right] \Upsilon_{2[h(t)]} \zeta(t) .
\end{gathered}
$$

With (18) and (19) and utilizing reciprocally convex optimization [31], if $\Omega=\left[\begin{array}{rl}\operatorname{diag}\left\{\mathbf{Q}_{1}, 3 \mathbf{Q}_{1}\right\} & \mathcal{S} \\ \mathcal{S}^{T} & \operatorname{diag}\left\{\mathbf{Q}_{2}, 3 \mathbf{Q}_{2}\right\}\end{array}\right]>0$, an upper bound of $\dot{V}_{5}(t)$ can be obtained as

$$
\begin{aligned}
\dot{V}_{5}(t)+ & x^{T}(t)\left(h_{U} Z_{1}\right) x(t) \\
& -x^{T}(t-h(t))\left(h_{U} Z_{1}\right) x(t-h(t))
\end{aligned}
$$

$$
\begin{aligned}
& -h_{U} \int_{t-h(t)}^{t}\left[\begin{array}{l}
x(t) \\
\dot{x}(t)
\end{array}\right]^{T}\left[\begin{array}{ll}
0_{n} & Z_{1} \\
Z_{1} & 0_{n}
\end{array}\right]\left[\begin{array}{l}
x(t) \\
\dot{x}(t)
\end{array}\right] d s \\
& +x^{T}(t-h(t))\left(h_{U} Z_{2}\right) x(t-h(t)) \\
& -x^{T}\left(t-h_{U}\right)\left(h_{U} Z_{2}\right) x\left(t-h_{U}\right) \\
& -h_{U} \int_{t-h_{U}}^{t-h(t)}\left[\begin{array}{l}
x(t) \\
\dot{x}(t)
\end{array}\right]^{T}\left[\begin{array}{ll}
0_{n} & Z_{2} \\
Z_{2} & 0
\end{array}\right]\left[\begin{array}{l}
x(t) \\
\dot{x}(t)
\end{array}\right] d s \\
& \leq \zeta^{T}(t)\left(\Xi_{5[h(t)]}-\left[\begin{array}{l}
\Upsilon_{1[h(t)]} \\
\Upsilon_{2[h(t)]}
\end{array}\right]^{T} \Omega\left[\begin{array}{l}
\Upsilon_{1[h(t)]} \\
\Upsilon_{2[h(t)]}
\end{array}\right]\right) \zeta(t) .
\end{aligned}
$$

In addition, from (6), for any positive diagonal matrices $H_{i}=\operatorname{diag}\left\{h_{1 i}, \ldots, h_{n i}\right\}(i=1, \ldots, 3)$, the following inequality holds:

$$
\begin{aligned}
& 0 \leq-2 \sum_{i=1}^{n} h_{i 1} {\left[f_{i}\left(W_{2, i} x(t)\right)-k_{i}^{-} W_{2, i} x(t)\right] } \\
& \times {\left[f_{i}\left(W_{2, i} x(t)\right)-k_{i}^{+} W_{2, i} x(t)\right] } \\
&-2 \sum_{i=1}^{n} h_{i 2}\left[f_{i}\left(W_{2, i} x(t-h(t))\right)-k_{i}^{-} W_{2, i} x(t-h(t))\right] \\
& \times\left[f_{i}\left(W_{2, i} x(t-h(t))\right)-k_{i}^{+} W_{2, i} x(t-h(t))\right] \\
&-2 \sum_{i=1}^{n} h_{i 3}\left[f_{i}\left(W_{2, i} x\left(t-h_{U}\right)\right)-k_{i}^{-} W_{2, i} x\left(t-h_{U}\right)\right] \\
& \times\left[f_{i}\left(W_{2, i} x\left(t-h_{U}\right)\right)-k_{i}^{+} W_{2, i} x\left(t-h_{U}\right)\right] \\
&=\zeta^{T}(t) \Theta_{1} \zeta(t) .
\end{aligned}
$$

Also, inspired by the authors' work of [7], from (5), the following conditions hold:

$$
\begin{array}{r}
k_{i}^{-} \leq \frac{f_{i}\left(W_{2, i} x(t)\right)-f_{i}\left(W_{2, i} x(t-h(t))\right)}{W_{2, i} x(t)-W_{2, i} x(t-h(t))} \leq k_{i}^{+}, \\
k_{i}^{-} \leq \frac{f_{i}\left(W_{2, i} x(t-h(t))\right)-f_{i}\left(W_{2, i} x\left(t-h_{U}\right)\right)}{W_{2, i} x(t-h(t))-W_{2, i} x\left(t-h_{U}\right)} \leq k_{i}^{+}, \\
i=1, \ldots, n .
\end{array}
$$

Therefore, for any positive diagonal matrices $H_{i}=\operatorname{diag}\left\{h_{1 i}\right.$, $\left.\ldots, h_{n i}\right\}(i=4,5)$, the following inequality holds: 


$$
\begin{aligned}
& 0 \leq-2 \sum_{i=1}^{n}\left\{h_{i 4}[\right. f_{i}\left(W_{2, i} x(t)\right)-f_{i}\left(W_{2, i} x(t-h(t))\right) \\
&-\left.k_{i}^{-}\left(W_{2, i} x(t)-W_{2, i} x(t-h(t))\right)\right] \\
& \times f_{i}\left(W_{2, i} x(t)\right)-f_{i}\left(W_{2, i} x(t-h(t))\right) \\
&-\left.\left.k_{i}^{+}\left(W_{2, i} x(t)-W_{2, i} x(t-h(t))\right)\right]\right\} \\
&-2 \sum_{i=1}^{n}\left\{h _ { i 5 } \left[f_{i}\left(W_{2, i} x(t-h(t))\right)-f_{i}\left(W_{2, i} x\left(t-h_{U}\right)\right)\right.\right. \\
&\left.-k_{i}^{-}\left(W_{2, i} x(t-h(t))-W_{2, i} x\left(t-h_{U}\right)\right)\right] \\
& \times\left[f_{i}\left(W_{2, i} x(t-h(t))\right)-f_{i}\left(W_{2, i} x\left(t-h_{U}\right)\right)\right. \\
&\left.\left.-k_{i}^{+}\left(W_{2, i} x(t-h(t))-W_{2, i} x\left(t-h_{U}\right)\right)\right]\right\} \\
&=\zeta^{T}(t) \Theta_{2} \zeta(t) .
\end{aligned}
$$

From (12)-(23) and by application of the S-procedure [46], an upper bound of $\dot{V}(t)$ can be written as

$\dot{V}(t)$

$\leq \zeta^{T}(t) \underbrace{\left(\Xi_{1[h(t)]}+\Xi_{2}+\Xi_{3}+\Xi_{4[h(t)]}+\Xi_{5[h(t)]}+\Theta_{1}+\Theta_{2}\right)}_{\Sigma_{[h(t)]}} \zeta(t)$

$$
\begin{aligned}
& -\zeta^{T}(t)\left[\begin{array}{l}
\Psi_{[h(t)]} \\
\Upsilon_{1[h(t)]} \\
\Upsilon_{2[h(t)]}
\end{array}\right]^{T}\left[\begin{array}{ll|l}
\left(1-h_{D}\right) \mathscr{G}_{1} & 0_{3 n \cdot 12 n} \\
\hline 0_{12 n \cdot 3 n} & \Omega
\end{array}\right] \\
& \times \underbrace{\left[\begin{array}{l}
\Psi_{[h(t)]} \\
\Upsilon_{1[h(t)]} \\
\Upsilon_{2[h(t)]}
\end{array}\right] \zeta(t) .}_{\$_{[h(t)]}}
\end{aligned}
$$

By Lemma 4, the condition

$$
\begin{aligned}
& \zeta^{T}(t)\left(\Sigma_{[h(t)]}-\$_{[h(t)]}^{T}\left[\begin{array}{l|l|l}
\left(1-h_{D}\right) \mathscr{G}_{1} & 0_{3 n \cdot 12 n} \\
\hline 0_{12 n \cdot 3 n} & \Omega
\end{array}\right] \$_{[h(t)]}\right) \zeta(t) \\
& \quad<0
\end{aligned}
$$

with $0=\Gamma \zeta(t)$ is equivalent to

$$
\begin{aligned}
& \left(\Gamma^{\perp}\right)^{T}\left(\Sigma_{[h(t)]}-\$_{[h(t)]}^{T}\left[\begin{array}{l|l}
\left(1-h_{D}\right) \mathscr{G}_{1} & 0_{3 n \cdot 12 n} \\
\hline 0_{12 n \cdot 3 n} & \Omega
\end{array}\right] \$_{[h(t)]}\right) \Gamma^{\perp} \\
& \quad<0 .
\end{aligned}
$$

Then, by Lemma 5, the condition (26) is equivalent to the following inequality with any matrix $F \in \mathbb{R}^{15 n \times 11 n}$ :

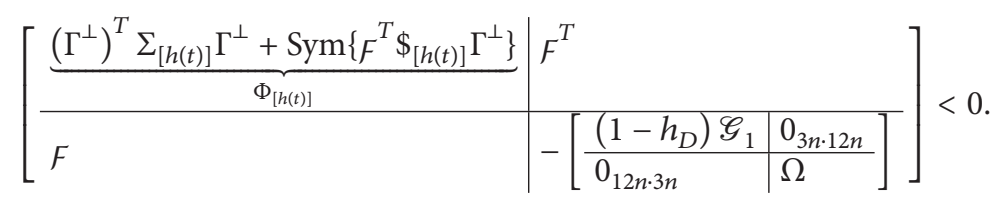

The above condition is affinely dependent on $h(t)$. Therefore, if inequalities (10) and (11) hold, then inequality (27) is satisfied, which means that system (4) is asymptotically stable for $0 \leq h(t) \leq h_{U}$ and $\dot{h}(t) \leq h_{D}$. It should be noted that $\left[\begin{array}{l|l}\left(1-h_{D}\right) \mathscr{G}_{1} & 0_{3 n \cdot 12 n} \\ \hline 0_{12 n \cdot 3 n} & \Omega\end{array}\right]>0$ holds if inequalities (11) and (12) are feasible. This completes our proof.

Remark 7. Theorem 6 utilized $V_{5}(t)$ which was inspired by the author's work [22]. In [22], the problem of delaydependent stability for neural networks with interval timevarying delays was addressed. However, when estimating the time-derivative of value obtained by $V_{5}(t)$, Wirtinger-based integral inequality has not been used in [22], which means that there is still room for further improvement in enhancing the feasible region of stability condition. In (18) and (19), the integral terms

$$
-h_{U} \int_{t-h(t)}^{t}\left[\begin{array}{c}
\dot{x}(s) \\
x(s) \\
\int_{s}^{t} \dot{x}(u) d u
\end{array}\right]^{T} \mathbf{Q}_{1}\left[\begin{array}{c}
\dot{x}(s) \\
x(s) \\
\int_{s}^{t} \dot{x}(u) d u
\end{array}\right] d s
$$

$$
-h_{U} \int_{t-h_{U}}^{t-h(t)}\left[\begin{array}{c}
\dot{x}(s) \\
x(s) \\
\int_{s}^{t} \dot{x}(u) d u
\end{array}\right]^{T} \mathbf{Q}_{2}\left[\begin{array}{c}
\dot{x}(s) \\
x(s) \\
\int_{s}^{t} \dot{x}(u) d u
\end{array}\right] d s
$$

were estimated by the use of Lemma 3 for the first time. Thus, more cross terms such as

$$
\begin{aligned}
& -\frac{h_{U}}{h(t)}\left[\begin{array}{c}
-x(t)-x(t-h(t))+2 v_{1}(t) \\
h(t) v_{1}(t)-2 v_{3}(t) \\
2 v_{3}(t)-h(t) v_{1}(t)
\end{array}\right]^{T} \\
& \times\left(3 \mathbf{Q}_{1}\right)\left[\begin{array}{c}
-x(t)-x(t-h(t))+2 v_{1}(t) \\
h(t) v_{1}(t)-2 v_{3}(t) \\
2 v_{3}(t)-h(t) v_{1}(t)
\end{array}\right] \\
& -\frac{h_{U}}{h_{U}-h(t)}\left[\begin{array}{c}
-x(t-h(t))-x\left(t-h_{U}\right)+2 v_{2}(t) \\
\left(h_{U}-h(t)\right) v_{2}(t)-2 v_{4}(t) \\
2 v_{4}(t)-\left(h_{U}-h(t)\right) v_{2}(t)
\end{array}\right]^{T}
\end{aligned}
$$




$$
\times\left[\begin{array}{c}
-x(t-h(t))-x\left(t-h_{U}\right)+2 v_{2}(t) \\
\left(h_{U}-h(t)\right) v_{2}(t)-2 v_{4}(t) \\
2 v_{4}(t)-\left(h_{U}-h(t)\right) v_{2}(t)
\end{array}\right]
$$

were utilized in the stability condition of Theorem 6 .

Remark 8. It should be noted that when $h_{D}$ is larger than one, the condition presented in Theorem 6 is infeasible since the term $-\left(1-h_{D}\right) \mathscr{G}$ cannot be negative. When $h_{D}$ is unknown or larger than 1 , then, by not considering $V_{4}(t)$ of the functional in (12), the following corollary can be obtained.

Remark 9. In Lemma 3 , the term $(2 /(b-a)) \int_{a}^{b} \int_{s}^{b} \omega(u) d u d s$ is included in (7) instead of $(2 /(b-a)) \int_{a}^{b} \int_{a}^{s} \omega(u) d u d s$ in [32]. Thus, the integral terms such as $v_{3}(t)$ and $v_{4}(t)$ of Theorem 6 can be utilized as the element of the augmented vector $\zeta(t)$.

Corollary 10. For given scalars $h_{U}>0$, and diagonal matrices $K_{m}=\operatorname{diag}\left\{k_{1}^{-}, \ldots, k_{n}^{-}\right\}$and $K_{p}=\operatorname{diag}\left\{k_{1}^{+}, \ldots, k_{n}^{+}\right\}$, system (4) is asymptotically stable for $0 \leq h(t) \leq h_{U}$, if there exist matrices $\Lambda=\operatorname{diag}\left\{\lambda_{1}, \ldots, \lambda_{n}\right\} \in \mathbb{S}_{+}^{n}, \Delta=\operatorname{diag}\left\{\delta_{1}, \ldots, \delta_{n}\right\} \in \mathbb{S}_{+}^{n}, H_{i}=$ $\operatorname{diag}\left\{h_{1 i}, \ldots, h_{n i}\right\} \in \mathbb{S}_{+}^{n}(i=1,2, \ldots, 5), \mathscr{R} \in \mathbb{S}_{+}^{3 n}, \mathcal{N} \in \mathbb{S}_{+}^{3 n}$, $Q \in \mathbb{S}_{+}^{3 n}, \mathcal{S} \in \mathbb{R}^{6 n \times 6 n}, \hat{F} \in \mathbb{R}^{12 n \times 11 n}, Z_{i} \in \mathbb{S}^{n}(i=1,2)$ satisfying the following LMIs:

$$
\begin{aligned}
& {\left[\begin{array}{l|l}
\Phi_{[h(t)=0]} & \widehat{F}^{T} \\
\hline \widehat{F} & -\Omega
\end{array}\right]<0,} \\
& {\left[\begin{array}{l|l}
\Phi_{\left[h(t)=h_{U}\right]} & \hat{F}^{T} \\
\hline \widehat{F} & -\Omega
\end{array}\right]<0,}
\end{aligned}
$$

where $\Phi_{h(t)}$ and $\Omega$ are defined in the appendix with $G_{2}=0_{n}$.

Proof. The proof of Corollary 10 is very similar to the proof of Theorem 6 . Thus, it is omitted.

\section{Numerical Examples}

In this section, two numerical examples are introduced to show the improvements of the proposed methods.

Example 1. Consider the neural networks (4) with the parameters

$$
\begin{gathered}
A=\operatorname{diag}\{2,2\} \\
W_{0}=\left[\begin{array}{cc}
1 & 1 \\
-1 & -1
\end{array}\right], \quad W_{1}=\left[\begin{array}{cc}
0.88 & 1 \\
1 & 1
\end{array}\right], \quad W_{2}=I_{2 n}, \\
K_{p}=\operatorname{diag}\{0.4,0.8\}, \quad K_{m}=\operatorname{diag}\{0,0\} .
\end{gathered}
$$

This example has been utilized in many previous works to check the conservatism of delay-dependent stability. In Table 1, the comparison of our results with the previous works of [7-9], and [10-12] are conducted. When $h_{D}$ is 0.8 or 0.9 , Theorem 6 provides significantly larger delay bounds than those listed in Table 1. Also, When $h_{D}$ is larger than one or unknown, the result of Corollary 10 is the largest value
TABLE 1: Delay bounds $h_{U}$ with different $h_{D}$ (Example 1).

\begin{tabular}{lccc}
\hline Method & \multicolumn{2}{c}{$h_{D}$} \\
& 0.8 & 0.9 & unknown or $\geq 1$ \\
\hline$[10](m=2)^{*}$ & 2.8794 & 1.9562 & 1.7768 \\
{$[11](m=2)^{*}$} & 2.8980 & 1.9562 & 1.7770 \\
{$[9](m=2)^{*}$} & 2.8991 & 2.0087 & - \\
{$[8](m=2)^{*}$} & 2.9541 & 1.9654 & 1.7839 \\
{$[7]$} & 3.7174 & 2.8339 & 2.8222 \\
{$[12]$} & 4.5940 & 3.4671 & 3.4504 \\
{$[34]$} & 5.0945 & 3.4978 & 3.4506 \\
Theorem 6 & 6.1889 & 3.7300 & - \\
Corollary 10 & - & - & 3.4702 \\
\hline * & & &
\end{tabular}

${ }^{*} m$ is delay-partitioning number.

TABLE 2: Upper bounds of time-varying delays with different $h_{D}$ (Example 2).

\begin{tabular}{lcccc}
\hline Methods & $h_{D}=0.0$ & $h_{D}=0.1$ & $h_{D}=0.5$ & $h_{D}$ is unknown \\
\hline$[13]$ & 1.3323 & 0.8246 & 0.3733 & 0.2313 \\
{$[14]$} & 1.3323 & 0.8402 & 0.4264 & 0.3209 \\
{$[15]$} & 1.3323 & 0.8402 & 0.4264 & 0.3209 \\
{$[17]$} & - & 0.8411 & 0.4267 & 0.3215 \\
$[16]$ (Th. 1, $N=2)$ & 1.5330 & 0.9331 & 0.4268 & 0.3215 \\
{$[33]$ Cor. 1) } & 1.5575 & 0.9430 & 0.4417 & 0.3632 \\
Theorem 6 & 1.8899 & 1.1174 & 0.4541 & - \\
Corollary 10 & - & - & - & 0.3936 \\
\hline
\end{tabular}

comparing with those in $[7,8,10-34]$. Therefore, the result of Table 1 shows that the proposed Theorem 6 and Corollary 10 effectively reduce the conservatism of stability criteria.

Example 2. Consider the static recurrent neural networks where

$$
\begin{gathered}
A=\operatorname{diag}\{7.3458,6.9987,5.5949\}, \\
W_{0}=I_{3 n}, \quad W_{1}=I_{3 n}, \\
W_{2}=\left[\begin{array}{ccc}
13.6014 & -2.9616 & -0.6936 \\
7.4736 & 21.6810 & 3.2100 \\
0.7290 & -2.6334 & -20.1300
\end{array}\right], \\
K_{p}=\operatorname{diag}\{0.3680,0.1795,0.2876\}, \quad K_{m}=0_{3 n} .
\end{gathered}
$$

The maximum delay bounds obtained by Theorem 6 and Corollary 10 and the results of [13-33] are listed in Table 2. From this table, it can be confirmed that the proposed stability condition also gives larger delay bounds, which support the less conservatism of Theorem 6 and Corollary 10.

\section{Conclusion}

In this paper, two improved delay-dependent stability criteria for generalized neural networks with time-varying delays have been proposed by the use of the augmented Lyapunov stability theorem and Lemma 3 . In Theorem 6 , by constructing the suitable augmented Lyapunov-Krasovskii functional 
and utilizing Lemma 3, the delay-dependent sufficient condition for asymptotic stability of the concerned network was derived. When $h_{D}$ is larger than one or unknown, the stability condition was also presented as Corollary 10 based on the result of Theorem 6. Via two numerical examples which have been dealt with in many previous works to check the conservatism of stability criteria, it was shown that Theorem 6 and Corollary 10 provide larger delay bounds than those of the recent works.

\section{Appendix}

Consider

$$
\begin{aligned}
& v_{1}(t)=\frac{1}{h(t)} \int_{t-h(t)}^{t} x(s) d s, \\
& v_{2}(t)=\frac{1}{h_{U}-h(t)} \int_{t-h_{U}}^{t-h(t)} x(s) d s, \\
& v_{3}(t)=\frac{1}{h(t)} \int_{t-h(t)}^{t} \int_{s}^{t} x(u) d u d s, \\
& v_{4}(t)=\frac{1}{h_{U}-h(t)} \int_{t-h_{U}}^{t-h(t)} \int_{s}^{t} x(u) d u d s, \\
& \zeta(t)=\operatorname{col}\left\{x(t), x(t-h(t)), x\left(t-h_{U}\right), \dot{x}(t), \dot{x}\left(t-h_{U}\right)\right. \text {, } \\
& v_{1}(t), v_{2}(t), v_{3}(t), v_{4}(t), f\left(W_{2} x(t)\right), \\
& \left.f\left(W_{2} x(t-h(t))\right), f\left(W_{2} x\left(t-h_{U}\right)\right)\right\}, \\
& \Gamma=\left[-A,-I_{n}, W_{0}, W_{1}\right]\left[e_{1}, e_{4}, e_{10}, e_{11}\right]^{T}, \\
& \Xi_{1[h(t)]} \\
& =\operatorname{Sym}\left\{\left[e_{1}, e_{3}, h(t) e_{6}+(h-h(t)) e_{7}\right] \mathscr{R}\left[e_{4}, e_{5}, e_{1}-e_{3}\right]^{T}\right\}, \\
& \Xi_{2}=\operatorname{Sym}\left\{\left[e_{10}-e_{1} W_{2}^{T} K_{m}\right] \Lambda W_{2} e_{4}^{T}\right\} \\
& +\operatorname{Sym}\left\{\left[e_{1} W_{2}^{T} K_{p}-e_{10}\right] \Delta W_{2} e_{4}^{T}\right\}, \\
& \Xi_{3}=\left[e_{1}, e_{4}, e_{10}\right] \mathcal{N}\left[e_{1}, e_{4}, e_{10}\right]^{T} \\
& -\left[e_{3}, e_{5}, e_{12}\right] \mathcal{N}\left[e_{3}, e_{5}, e_{12}\right]^{T}, \\
& \Xi_{4[h(t)]}=\left[e_{1}, 0_{12 n \cdot n}, 0_{12 n \cdot n}\right] \mathscr{G}_{1}\left[e_{1}, 0_{12 n \cdot n}, 0_{12 n \cdot n}\right]^{T} \\
& +h(t) \operatorname{Sym}\left\{\left[e_{6}, e_{1}-e_{6}, e_{8}\right] \mathscr{G}_{1}\left[0_{12 n \cdot n}, e_{4}, e_{1}\right]^{T}\right\} \\
& +e_{10} G_{2} e_{10}^{T}-\left(1-h_{D}\right) e_{11} G_{2} e_{11}^{T} \text {, } \\
& \mathbf{Q}_{i}=Q+\left[\begin{array}{lll}
0_{n} & Z_{i} & 0_{n} \\
Z_{i} & 0_{n} & 0_{n} \\
0_{n} & 0_{n} & 0_{n}
\end{array}\right] \quad(i=1,2) \\
& \Omega=\left[\begin{array}{cc}
\operatorname{diag}\left\{\mathbf{Q}_{1}, 3 \mathbf{Q}_{1}\right\} & \mathcal{S} \\
\mathcal{S}^{T} & \operatorname{diag}\left\{\mathbf{Q}_{2}, 3 \mathbf{Q}_{2}\right\}
\end{array}\right], \\
& \Pi_{[h(t)]}=\left[\begin{array}{c}
h_{U} e_{1}^{T}-h(t) e_{6}^{T}-\left(h_{U}-h(t)\right) e_{7}^{T} \\
h(t) e_{8}^{T}+\left(h_{U}-h(t)\right) e_{9}^{T} \\
\left(\frac{h_{U}^{2}}{2}\right) e_{1}^{T}-h(t) e_{8}^{T}-\left(h_{U}-h(t)\right) e_{9}^{T}
\end{array}\right], \\
& \Xi_{5[h(t)]}=h_{U}^{2}\left[e_{4}, e_{1}, 0_{12 n \cdot n}\right] \mathbb{Q}\left[e_{4}, e_{1}, 0_{12 n \cdot n}\right]^{T}
\end{aligned}
$$

$$
\begin{aligned}
& +h_{U} \operatorname{Sym}\left\{\Pi_{[h(t)]}^{T} Q\left[0_{12 n \cdot n}, 0_{12 n \cdot n}, e_{4}\right]^{T}\right\} \\
& +h_{U}\left\{e_{1} Z_{1} e_{1}^{T}-e_{2} Z_{1} e_{2}^{T}+e_{2} Z_{2} e_{2}^{T}-e_{3} Z_{2} e_{3}^{T}\right\}, \\
& \Theta_{1}=-\operatorname{Sym}\left\{\left(e_{10}-e_{1} W_{2}^{T} K_{m}\right) H_{1}\left(e_{10}-e_{1} W_{2}^{T} K_{p}\right)^{T}\right\} \\
& -\operatorname{Sym}\left\{\left(e_{11}-e_{2} W_{2}^{T} K_{m}\right) H_{2}\left(e_{11}-e_{2} W_{2}^{T} K_{p}\right)^{T}\right\} \\
& -\operatorname{Sym}\left\{\left(e_{12}-e_{3} W_{2}^{T} K_{m}\right) H_{3}\left(e_{12}-e_{3} W_{2}^{T} K_{p}\right)^{T}\right\}, \\
& \Theta_{2}=-\operatorname{Sym}\left\{\left(e_{10}-e_{11}-\left(e_{1}-e_{2}\right) W_{2}^{T} K_{m}\right) H_{4}\right. \\
& \left.\times\left(e_{10}-e_{11}-\left(e_{1}-e_{2}\right) W_{2}^{T} K_{p}\right)^{T}\right\} \\
& -\operatorname{Sym}\left\{\left(e_{11}-e_{12}-\left(e_{2}-e_{3}\right) W_{2}^{T} K_{m}\right) H_{5}\right. \\
& \left.\times\left(e_{11}-e_{12}-\left(e_{2}-e_{3}\right) W_{2}^{T} K_{p}\right)^{T}\right\}, \\
& \Sigma_{[h(t)]}=\Xi_{1[h(t)]}+\Xi_{2}+\Xi_{3}+\Xi_{4[h(t)]} \\
& +\Xi_{5[h(t)]}+\Theta_{1}+\Theta_{2}, \\
& \Psi_{[h(t)]}=\left[e_{2}, e_{1}-e_{2}, h(t) e_{6}\right]^{T}, \\
& \Upsilon_{1[h(t)]}=\left[\begin{array}{c}
e_{1}^{T}-e_{2}^{T} \\
h(t) e_{6}^{T} \\
h(t)\left(e_{1}-e_{6}\right)^{T} \\
-e_{1}^{T}-e_{2}^{T}+2 e_{6}^{T} \\
h(t) e_{6}^{T}-2 e_{8}^{T} \\
2 e_{8}^{T}-h(t) e_{6}^{T}
\end{array}\right], \\
& \Upsilon_{2[h(t)]}=\left[\begin{array}{c}
e_{2}^{T}-e_{3}^{T} \\
\left(h_{U}-h(t)\right) e_{7}^{T} \\
\left(h_{U}-h(t)\right)\left(e_{1}-e_{7}\right)^{T} \\
-e_{2}^{T}-e_{3}^{T}+2 e_{7}^{T} \\
\left(h_{U}-h(t)\right) e_{7}^{T}-2 e_{9}^{T} \\
2 e_{9}^{T}-\left(h_{U}-h(t)\right) e_{7}^{T}
\end{array}\right] \text {, } \\
& \$_{[h(t)]}^{T}=\left[\Psi_{[h(t)]}^{T}, \Upsilon_{1[h(t)]}^{T}, \Upsilon_{2[h(t)]}^{T}\right], \\
& \Phi_{[h(t)]}=\left(\Gamma^{\perp}\right)^{T} \Sigma_{[h(t)]} \Gamma^{\perp}+\operatorname{Sym}\left\{F^{T} \$_{[h(t)]} \Gamma^{\perp}\right\} .
\end{aligned}
$$

\section{Conflict of Interests}

The authors declare that there is no conflict of interests regarding the publication of this paper. 


\section{Acknowledgments}

This research was supported by the Basic Science Research Program through the National Research Foundation of Korea (NRF) funded by the Ministry of Education, Science and Technology (2011-0009273). This work was also supported by the Human Resources Development of the Korea Institute of Energy Technology Evaluation and Planning (KETEP) grant funded by the Korea government Ministry of Trade, industry \& Energy (no. 20144030200450).

\section{References}

[1] S. Haykin, Neural Networks: A Comprehensive Foundation, Prentice-Hall, Englewood Cliffs, NJ, USA, 1998.

[2] G. Ou and Y. L. Murphey, "Multi-class pattern classification using neural networks," Pattern Recognition, vol. 40, no. 1, pp. $4-18,2007$.

[3] S. Xu, J. Lam, and D. W. C. Ho, "Novel global robust stability criteria for interval neural networks with multiple time-varying delays," Physics Letters Section A, vol. 342, no. 4, pp. 322-330, 2005.

[4] L. O. Chua and L. Yang, "Cellular neural networks: applications," IEEE Transactions on Circuits and Systems, vol. 35, no. 10, pp. 1273-1290, 1988.

[5] Q. Zhu, R. Rakkiyappan, and A. Chandrasekar, "Stochastic stability of Markovian jump BAM neural networks with leakage delays and impulse control," Neurocomputing, vol. 136, pp. 136151, 2014.

[6] Q. Zhu and J. Cao, "Exponential stability analysis of stochastic reaction-diffusion Cohen-Grossberg neural networks with mixed delays," Neurocomputing, vol. 74, no. 17, pp. 3084-3091, 2011.

[7] O. M. Kwon, J. H. Park, S. M. Lee, and E. J. Cha, "Analysis on delay-dependent stability for neural networks with timevarying delays," Neurocomputing, vol. 103, pp. 114-120, 2013.

[8] X. Zhou, J. Tian, H. Ma, and S. Zhong, "Improved delaydependent stability criteria for recurrent neural networks with time-varying delays," Neurocomputing, vol. 129, pp. 401-408, 2014.

[9] J. Tian, W. Xiong, and F. Xu, "Improved delay-partitioning method to stability analysis for neural networks with discrete and distributed time-varying delays," Applied Mathematics and Computation, vol. 233, pp. 152-164, 2014.

[10] H.-B. Zeng, Y. He, M. Wu, and C.-F. Zhang, "Complete delaydecomposing approach to asymptotic stability for neural networks with time-varying delays," IEEE Transactions on Neural Networks, vol. 22, no. 5, pp. 806-812, 2011.

[11] C. Ge, C. Hua, and X. Guan, "New delay-dependent stability criteria for neural networks with time-varying delay using delay-decomposition approach," IEEE Transactions on Neural Networks and Learning Systems, vol. 25, no. 7, pp. 1378-1383, 2014.

[12] O. M. Kwon, J. H. Park, S. M. Lee, and E. J. Cha, "New augmented Lyapunov-Krasovskii functional approach to stability analysis of neural networks with time-varying delays," Nonlinear Dynamics, vol. 76, no. 1, pp. 221-236, 2014.

[13] H. Shao, "Delay-dependent stability for recurrent neural networks with time-varying delays," IEEE Transactions on Neural Networks, vol. 19, no. 9, pp. 1647-1651, 2008.
[14] H. Shao, "Novel delay-dependent stability results for neural networks with time-varying delays," Circuits, Systems, and Signal Processing, vol. 29, no. 4, pp. 637-647, 2010.

[15] Z. Zuo, C. Yang, and Y. Wang, "A new method for stability analysis of recurrent neural networks with interval timevarying delay," IEEE Transactions on Neural Networks, vol. 21, no. 2, pp. 339-344, 2010.

[16] X. Li, H. Gao, and X. Yu, "A unified approach to the stability of generalized static neural networks with linear fractional uncertainties and delays," IEEE Transactions on Systems, Man, and Cybernetics, Part B: Cybernetics, vol. 41, no. 5, pp. 1275-1286, 2011.

[17] Y.-Q. Bai and J. Chen, "New stability criteria for recurrent neural networks with interval time-varying delay," Neurocomputing, vol. 121, pp. 179-184, 2013.

[18] K. Gu, "A further refinement of discretized Lyapunov functional method for the stability of time-delay systems," International Journal of Control, vol. 74, no. 10, pp. 967-976, 2001.

[19] Y. Ariba and F. Gouaisbaut, "An augmented model for robust stability analysis of time-varying delay systems," International Journal of Control, vol. 82, no. 9, pp. 1616-1626, 2009.

[20] J. Sun, G. P. Liu, and J. Chen, "Delay-dependent stability and stabilization of neutral time-delay systems," International Journal of Robust and Nonlinear Control, vol. 19, no. 12, pp. 13641375, 2009.

[21] J. Sun, G. P. Liu, J. Chen, and D. Rees, "Improved delay-rangedependent stability criteria for linear systems with time-varying delays," Automatica, vol. 46, no. 2, pp. 466-470, 2010.

[22] O. M. Kwon, M. J. Park, J. H. Park, S. M. Lee, and E. J. Cha, "On stability analysis for neural networks with interval timevarying delays via some new augmented Lyapunov-Krasovskii functional," Communications in Nonlinear Science and Numerical Simulation, vol. 19, no. 9, pp. 3184-3201, 2014.

[23] Y. He, Q.-G. Wang, C. Lin, and M. Wu, "Augmented Lyapunov functional and delay-dependent stability criteria for neutral systems," International Journal of Robust and Nonlinear Control, vol. 15, no. 18, pp. 923-933, 2005.

[24] M. N. Alpaslan Parlakçi, "Extensively augmented Lyapunov functional approach for the stability of neutral time-delay systems," IET Control Theory \& Applications, vol. 2, no. 5, pp. 431-436, 2008.

[25] P. Park, "A delay-dependent stability criterion for systems with uncertain time-invariant delays," IEEE Transactions on Automatic Control, vol. 44, no. 4, pp. 876-877, 1999.

[26] D. Yue, S. Won, and O. Kwon, "Delay dependent stability of neutral systems with time delay: an LMI approach," IEE Proceedings: Control Theory and Applications, vol. 150, no. 1, pp. 23-27, 2003.

[27] O. M. Kwon and J. H. Park, "On improved delay-dependent robust control for uncertain time-delay systems," IEEE Transactions on Automatic Control, vol. 49, no. 11, pp. 1991-1995, 2004.

[28] X.-G. Liu, M. Wu, R. Martin, and M.-L. Tang, "Stability analysis for neutral systems with mixed delays," Journal of Computational and Applied Mathematics, vol. 202, no. 2, pp. 478-497, 2007.

[29] Y. He, G. P. Liu, D. Rees, and M. Wu, "Stability analysis for neural networks with time-varying interval delay," IEEE Transactions on Neural Networks, vol. 18, no. 6, pp. 1850-1854, 2007.

[30] P. Park and J. W. Ko, "Stability and robust stability for systems with a time-varying delay," Automatica, vol. 43, no. 10, pp. 1855$1858,2007$. 
[31] P. Park, J. W. Ko, and C. Jeong, "Reciprocally convex approach to stability of systems with time-varying delays," Automatica, vol. 47, no. 1, pp. 235-238, 2011.

[32] A. Seuret and F. Gouaisbaut, "Wirtinger-based integral inequality: application to time-delay systems," Automatica, vol. 49, no. 9, pp. 2860-2866, 2013.

[33] M. D. Ji, Y. He, C. K. Zhang, and M. Wu, "Novel stability criteria for recurrent neural networks with time-varying delay," Neurocomputing, vol. 138, pp. 383-391, 2014.

[34] O. M. Kwon, M. J. Park, J. H. Park, S. M. Lee, and E. J. Cha, “On less conservative stability criteria for neural networks with timevarying delays utilizing Wirtinger-based integral inequality," Mathematical Problems in Engineering, vol. 2014, Article ID 859736, 13 pages, 2014.

[35] X.-M. Zhang and Q.-L. Han, "Global asymptotic stability for a class of generalized neural networks with interval time-varying delays," IEEE Transactions on Neural Networks, vol. 22, no. 8, pp. 1180-1192, 2011.

[36] C. K. Zhang, Y. He, L. Jiang, Q. H. Wu, and M. Wu, "Delaydependent stability criteria for generalized neural networks with two delay components," IEEE Transactions on Neural Networks and Learning Systems, vol. 25, no. 7, pp. 1263-1276, 2013.

[37] X. Li and S. Song, "Impulsive control for existence, uniqueness, and global stability of periodic solutions of recurrent neural networks with discrete and continuously distributed delays," IEEE Transactions on Neural Networks and Learning Systems, vol. 24, no. 6, pp. 868-877, 2013.

[38] X. Li and X. Fu, "Effect of leakage time-varying delay on stability of nonlinear differential systems," Journal of the Franklin Institute, vol. 350, no. 6, pp. 1335-1344, 2013.

[39] Q. Zhu and J. Cao, "Stability of Markovian jump neural networks with impulse control and time varying delays," Nonlinear Analysis. Real World Applications, vol. 13, no. 5, pp. 2259-2270, 2012.

[40] T. Li, W. X. Zheng, and C. Lin, "Delay-slope-dependent stability results of recurrent neural networks," IEEE Transactions on Neural Networks, vol. 22, no. 12, pp. 2138-2143, 2011.

[41] S. Xu, J. Lam, D. W. C. Ho, and Y. Zou, "Delay-dependent exponential stability for a class of neural networks with time delays," Journal of Computational and Applied Mathematics, vol. 183, no. 1, pp. 16-28, 2005.

[42] Y. Liu, Z. Wang, and X. Liu, "Global exponential stability of generalized recurrent neural networks with discrete and distributed delays," Neural Networks, vol. 19, no. 5, pp. 667-675, 2006.

[43] R. E. Skelton, T. Iwasaki, and K. M. Grigoradis, A Unified Algebraic Approach to Linear Control Design, Taylor \& Francis, New York, NY, USA, 1997.

[44] T. Wang, C. Zhang, S. Fei, and T. Li, "Further stability criteria on discrete-time delayed neural networks with distributed delay," Neurocomputing, vol. 111, pp. 195-203, 2013.

[45] S. H. Kim, P. Park, and C. Jeong, "Robust $H_{\infty}$ stabilisation of networked control systems with packet analyser," IET Control Theory and Applications, vol. 4, no. 9, pp. 1828-1837, 2010.

[46] S. Boyd, L. El Ghaoui, E. Feron, and V. Balakrishnan, Linear Matrix Inequalities in System and Control Theory, SIAM, Philadelphia, Pa, USA, 1994. 


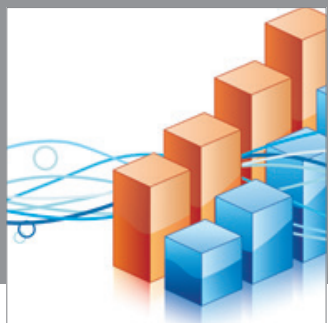

Advances in

Operations Research

mansans

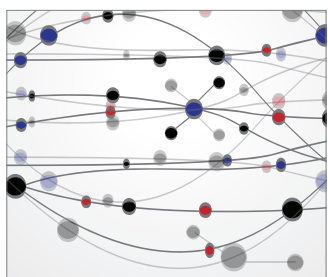

The Scientific World Journal
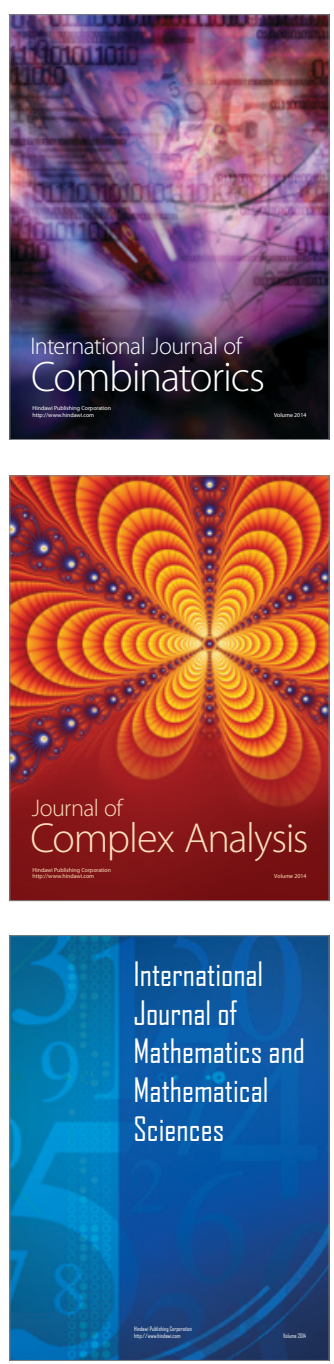
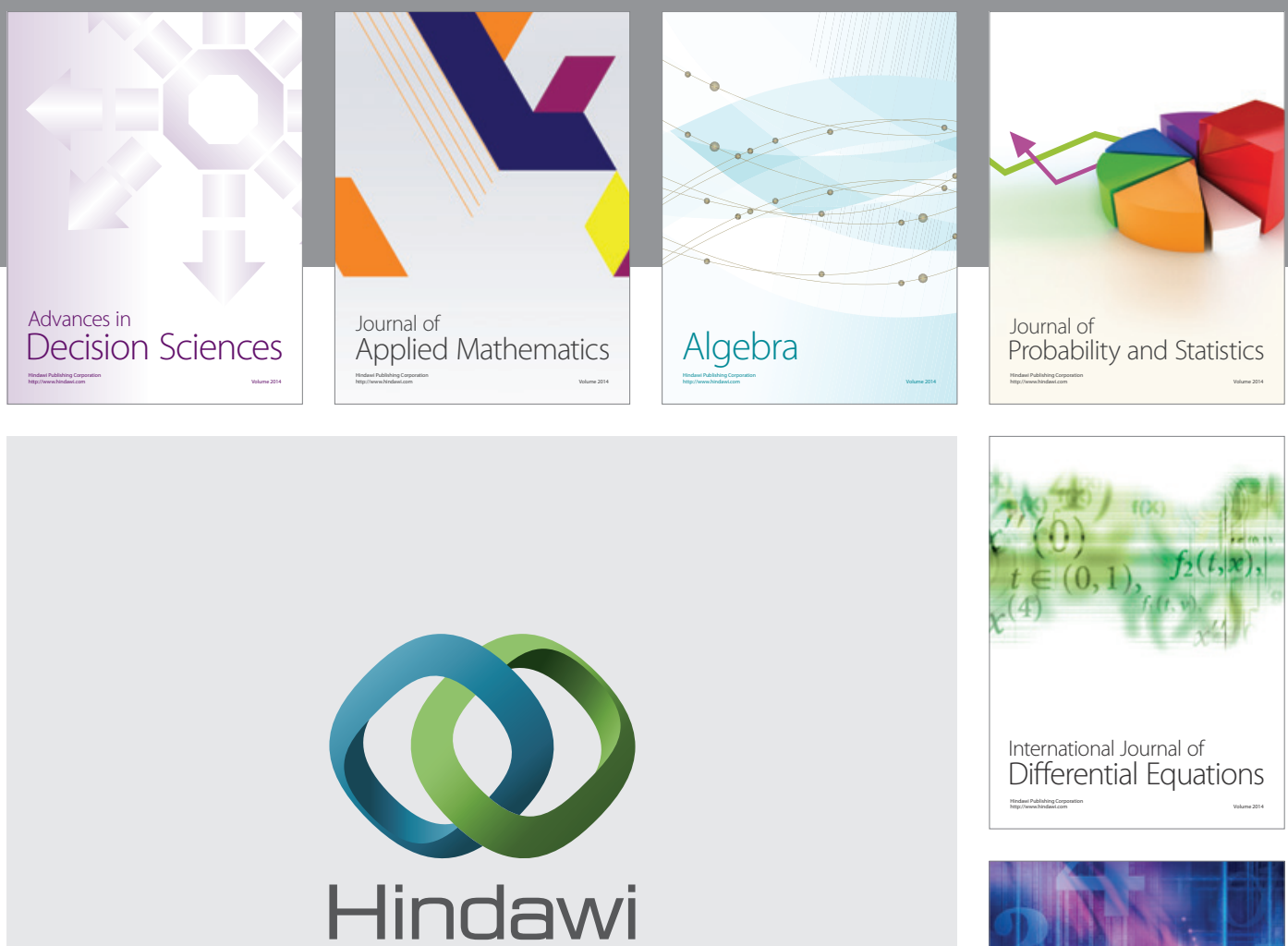

Submit your manuscripts at http://www.hindawi.com
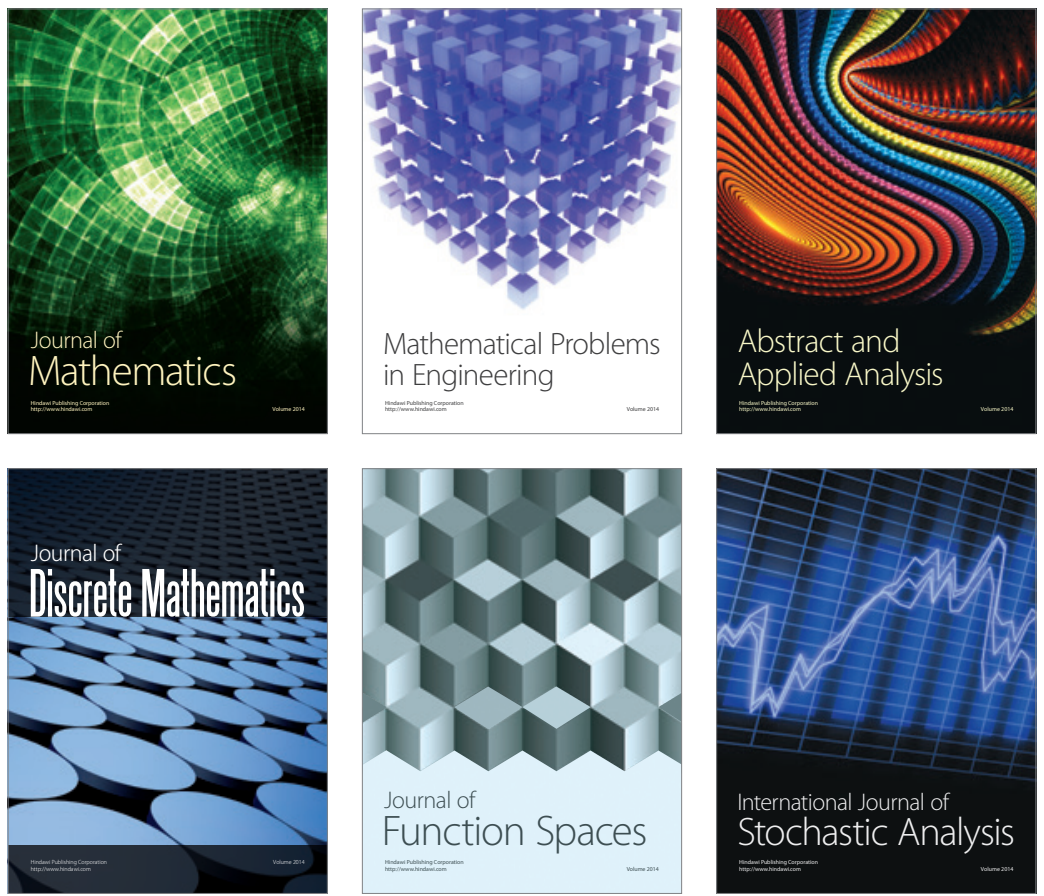

Journal of

Function Spaces

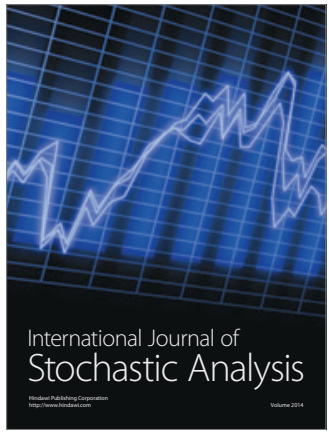

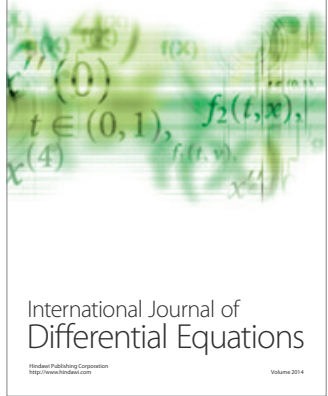
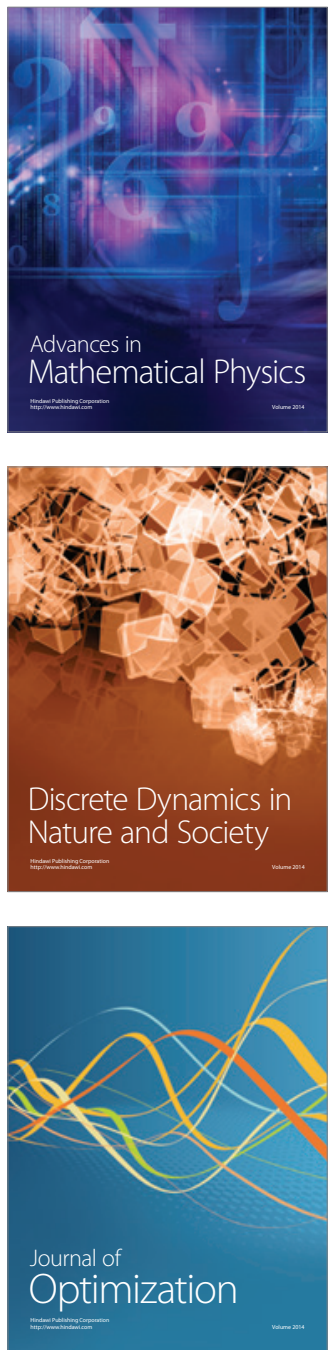